\title{
Approximate Next-to-Next-to-Leading Order Corrections to Hadronic Jet Production
}

\author{
Daniel de Florian, ${ }^{1}$ Patriz Hinderer, ${ }^{2}$ Asmita Mukherjee, ${ }^{3}$ Felix Ringer, ${ }^{2}$ and Werner Vogelsang ${ }^{2}$ \\ ${ }^{1}$ Departamento de Física, FCEyN, Universidad de Buenos Aires, (1428) Pabellón 1, Ciudad Universitaria, Capital Federal, Argentina \\ ${ }^{2}$ Institute for Theoretical Physics, Tübingen University, 72076 Tübingen, Germany \\ ${ }^{3}$ Department of Physics, Indian Institute of Technology Bombay, Powai, Mumbai 400076, India \\ (Received 27 October 2013; revised manuscript received 7 January 2014; published 25 February 2014)
}

\begin{abstract}
We determine dominant next-to-next-to-leading order QCD corrections to single-inclusive jet production at the LHC and Tevatron, using the established threshold resummation framework. In contrast to previous literature on this topic, our study incorporates all of the following features: (1) It properly accounts for the way a jet is defined in experiment and treated in available full next-to-leading order calculations, (2) it includes the three leading classes of logarithmic terms in the perturbative expansion, and (3) it is adapted to the full kinematics in jet transverse momentum and rapidity relevant for experiments. A recent full next-to-next-to-leading order calculation in the purely gluonic channel allows us to assess the region where our approximate corrections provide an accurate description. We expect our results to be important on the way to precision jet phenomenology at the LHC and as a benchmark for further full next-to-next-toleading order calculations.
\end{abstract}

DOI: 10.1103/PhysRevLett.112.082001

PACS numbers: 12.38.Bx, 13.85.-t, 13.87.-a

Introduction.-The production of high-transversemomentum hadron jets plays a fundamental role at the LHC [1] and at Tevatron [2]. Jets are produced very copiously, making them precision probes of the physics of the standard model and beyond. Theoretical calculations whose precision matches that achievable in experiment are of critical importance. The efforts made in this context have spanned more than three decades now, culminating, so far, with the recent calculation of the next-to-next-to-leading order (NNLO) perturbative corrections to jet production in the "gluon-only" channel $[3,4]$.

As complete NNLO calculations of jet production are probably still a few years away, it is useful to determine approximate NNLO results, at least in certain kinematical regimes. This is possible thanks to the fact that the perturbative series for the partonic cross sections contains classes of logarithmic terms that often dominate. Resummation techniques in QCD [5] allow us to determine the all-order structure of these logarithmic terms, and one, therefore, also obtains the logarithms present at NNLO. Knowledge of approximate NNLO expressions is very useful, since it potentially offers an avenue toward more precise phenomenology than available on the basis of the presently known full next-to-leading order (NLO) corrections. It also serves as a benchmark for future full NNLO calculations.

The logarithms just mentioned arise near a threshold from which the production of a jet becomes possible in a partonic collision. They are hence known as "threshold logarithms." The threshold is set by a vanishing invariant mass $\sqrt{s_{4}}$ of the partonic system that recoils against the observed jet. At the $k$ th order of perturbation theory, one finds threshold corrections to the Born cross section of the form $\alpha_{s}^{k}\left[\log ^{m}(z) / z\right]_{+}$, with $0 \leq m \leq 2 k-1$, where $z=$ $s_{4} / s$ with $\sqrt{s}$ the center-of-mass energy of the incoming partons. The systematic resummation of these logarithms to all orders in the strong coupling $\alpha_{s}$ was derived for the case of jet production in [5], where explicit next-to-leading logarithmic (NLL) results were given that in principle allow us to resum the three "towers" of logarithms with $m=2 k-1,2 k-2,2 k-3$.

An important "subtlety" was pointed out in [5] concerning the threshold logarithms in jet production: the structure of the logarithmic corrections depends on whether or not the jet is assumed to be massless at the partonic threshold, even at the leading-logarithmic (LL) level. If the jet is taken to be massless at threshold, an approach for which we will use the term "scheme (1)" in the following, LL corrections arise in the resummed perturbative function describing the jet. If, on the other hand, the jet is permitted to have a nonvanishing invariant mass at threshold ["scheme (2)"], the leading logarithms cancel, leaving behind a nonleading logarithm whose coefficient depends on jet "size" parameter $R$ introduced by the jet algorithm. The difference between the two schemes may be understood from the fact that fewer final states contribute in scheme (1) than in scheme (2) [5].

Approximate NNLO corrections for jet production have been derived in [6-8], adopting scheme (1). As one can see in the very recent study [7], the NLO terms predicted for scheme (1) fail to match a full NLO calculation [9] even in a regime where threshold logs are known to dominate. This becomes particularly evident from the fact that the threshold terms for scheme (1) do not carry any dependence on the jet parameter $R$, whereas the full NLO results do. These features observed in [7] are, in fact, not surprising: explicit 
analytical NLO calculations $[10,11]$ have shown that jets produced close to the partonic threshold do span a range of jet masses. Indeed, for any jet algorithm the jet produced in the perturbative calculation can evidently contain two or more partons and, hence, have a nonvanishing invariant mass. This is even the case at exact threshold $z=0$, when, for example, only a single parton recoils against the entire jet. The maximally allowed jet mass at threshold will depend on the parameter $R$ used in the jet algorithm.

Thus, the assumption of massless jets at threshold that was made in previous studies [6-8] does not appear to be appropriate. Instead, the resummation ought to be carried out within scheme (2). A resummed study in this scheme was, in fact, performed in [12], where, however, only the rapidity-integrated cross section was considered, for which the resummation simplifies considerably. Integration over all rapidity is not quite adequate for comparisons with experimental data. In the present Letter, we present new predictions for the NNLO threshold terms, using scheme (2) and keeping full dependence on rapidity in the calculation. We will also go beyond the previous studies $[6,7]$ by determining all three most leading logarithmic contributions $\propto\left(\log ^{3}(z) / z\right)_{+},\left(\log ^{2}(z) / z\right)_{+},(\log (z) / z)_{+}$at NNLO. The last of these is new; it may be obtained by matching the resummation framework to a full NLO calculation. For the latter we choose that of $[10,11]$, which provides analytical results for the partonic cross sections. The calculation was performed assuming that the produced jet is rather narrow ["narrow-jet approximation" (NJA)]. It has been shown that this approximation is extremely accurate even at relatively large jet sizes of $R \gtrsim 0.7$.

Theoretical framework.-The factorized cross section for the single-inclusive production of a jet with transverse momentum $p_{T}$ and pseudorapidity $\eta$ may be written as

$$
\begin{aligned}
\frac{p_{T}^{2} d^{2} \sigma}{d p_{T}^{2} d \eta}= & \sum_{a b} \int_{0}^{V(1-W)} d z \int_{V W /(1-z)}^{1-(1-V) /(1-z)} d v x_{a} f_{a}\left(x_{a}, \mu_{f}\right) \\
& \times x_{b} f_{b}\left(x_{b}, \mu_{f}\right) \frac{d \hat{\sigma}_{a b}}{d v d z}\left(v, z, p_{T}, \mu_{r}, \mu_{f}, R\right)
\end{aligned}
$$

where $\quad V=1-x_{T} e^{-\eta} / 2, \quad V W=x_{T} e^{\eta} / 2, \quad$ with $\quad x_{T}=$ $2 p_{T} / \sqrt{S}$ and the hadronic center-of-mass energy $\sqrt{S}$. The sum runs over all partonic collisions producing the jet; $d \hat{\sigma}_{a b}$ denote the corresponding partonic hard-scattering cross sections and $f_{a}, f_{b}$ the parton distribution functions at momentum fractions $x_{a}=V W / v(1-z), x_{b}=(1-V) /$ $(1-v)(1-z)$. The partonic cross sections are computed in QCD perturbation theory. As indicated, besides depending on $p_{T}$ and the usual renormalization and factorization scales $\mu_{r}, \mu_{f}$, they are functions of the partonic kinematic variables, which we have chosen as

$$
v=\frac{u}{t+u}, \quad z=\frac{s_{4}}{s},
$$

where $s=x_{a} x_{b} S$ is the partonic center-of-mass energy squared, $t=\left(p_{a}-p_{J}\right)^{2}, u=\left(p_{b}-p_{J}\right)^{2}$ (with $p_{a, b}$ and $p_{J}$ the four-momenta of the initial partons and the jet, respectively), and $s_{4}$ is the invariant mass squared of the "unobserved" partonic system recoiling against the jet. We stress that the $d \hat{\sigma}_{a b}$ also depend on the algorithm adopted to define the jet, as indicated by the generic jet parameter $R$ in Eq. (1). We always assume the jet to be defined by the anti$k_{t}$ algorithm [13].

The perturbative series for each of the partonic scattering cross sections may be cast into the form

$$
\frac{s d \hat{\sigma}_{a b}}{d v d z}=\left(\frac{\alpha_{s}}{\pi}\right)^{2}\left[\omega_{a b}^{(0)}+\frac{\alpha_{s}}{\pi} \omega_{a b}^{(1)}+\left(\frac{\alpha_{s}}{\pi}\right)^{2} \omega_{a b}^{(2)}+\mathcal{O}\left(\alpha_{s}^{3}\right)\right],
$$

where $\alpha_{s} \equiv \alpha_{s}(\mu)$ is the strong coupling constant, and where each of the $\omega_{a b}^{(k)}$ is a function of $v, z$ and, for $k>0$, of $R$ and $p_{T} / \mu$ (we choose, from now on, $\mu_{r}=\mu_{f} \equiv \mu$ ). At lowest order we have

$$
\omega_{a b}^{(0)}(v, z) \equiv \tilde{\omega}_{a b}^{(0)}(v) \delta(z),
$$

since the recoiling system is a single massless parton. Hence, $z=0$ sets a threshold for the process to take place, since the transverse momentum of the observed jet always needs to be balanced. At higher orders in perturbation theory, the hard scattering functions contain logarithmic distributions in $z$, with increasing powers of logarithms as the perturbative order increases. More precisely, one has near the threshold at $z=0$

$$
\alpha_{s}^{k} \omega_{a b}^{(k)} \sim \alpha_{s}^{k}\left(\frac{\log ^{m}(z)}{z}\right)_{+}, \text {with } 0 \leq m \leq 2 k-1 .
$$

Here, $\int_{0}^{1} d z g(z)[f(z)]_{+} \equiv \int_{0}^{1} d z[g(z)-g(0)] f(z)$. As one can see, two additional powers of the logarithm arise for every order of perturbation theory. Because of the integration against the parton distribution functions, which are steeply falling functions of momentum fraction, the threshold region $z \rightarrow 0$ typically makes significant contributions to the hadronic cross section. This is particularly the case when the kinematic boundary of the hadronic reaction is approached, that is, when $x_{T} \cosh \eta \rightarrow 1$.

As is well known, the large logarithmic corrections arising in the threshold region are associated with the emission of soft or collinear gluons. It is, therefore, possible to systematically determine the structure of the corrections to all orders and to resum the "towers" of logarithms with $m=2 k-1,2 k-2, \ldots$. This may be used to derive approximate beyond-NLO corrections for hadronic jet production, by expanding the resummed result appropriately to the desired order $[6,7,12]$. To achieve the all-order resummation, one considers Mellin moments in $(1-z)$ of the partonic cross section 


$$
\Omega_{a b}(v, N) \equiv \int_{0}^{1} d z(1-z)^{N-1} \frac{s d \hat{\sigma}_{a b}}{d v d z} .
$$

In moment space, the resummed hard-scattering function $\Omega_{a b}^{\text {res }}$ can, at large $N$, be written as $[5,14]$

$$
\begin{aligned}
\Omega_{a b}^{\mathrm{res}}(v, N)= & \sum_{c, d} \Delta_{a}\left(N_{a}\right) \Delta_{b}\left(N_{b}\right) J_{c}^{(\mathrm{jet})}(N, R) J_{d}^{(\mathrm{recoil})}(N) \\
& \times \Delta_{a b \rightarrow c d}^{(\mathrm{int})}(N, v) \Delta_{c}^{(\mathrm{ng})}(N),
\end{aligned}
$$

where $N_{a}=v N, N_{b}=(1-v) N$ and the sum runs over the two final-state partons $c, d$ in an underlying $a b \rightarrow c d$ subprocess. Here it is assumed that parton $c$ produces the jet (in a way that we shall clarify below), while the recoiling parton $d$ remains unobserved. Each of the terms is also a function of $\alpha_{s}(\mu)$ and $\log \left(\mu^{2} / s\right)$, which we have not written explicitly. Each of the functions $\Delta_{a}, \Delta_{b}, J_{c}^{\text {(jet) }}, J_{d}^{\text {(recoil) }}$ is an exponential. $\Delta_{a}, \Delta_{b}$ resum threshold logarithms arising from soft or collinear radiation off the incoming hard partons. Their expressions are very well known and may be found in the form we need them in, for example, [14]. Likewise, also the expression for gluon radiation off the "unobserved" recoiling parton $d$ is standard and may be found there. $\Delta_{a}, \Delta_{b}$, and $J_{d}^{\text {(recoil) }}$ contain all the leading logarithmic pieces $\propto\left(\log ^{3}(z) / z\right)_{+},\left(\log ^{2}(z) / z\right)_{+}$in $\omega_{a b}^{(2)}$.

A crucial point of our study concerns the function $J_{c}^{\text {(jet) }}$ used for the actual jet. As was shown in [5], this function takes different forms depending on whether one assumes the jet to become itself massless at threshold or not. These two forms differ even at leading logarithmic level. For scheme (2) introduced earlier, we have to next-toleading logarithmic accuracy [5]

$$
\log J_{c}^{(\mathrm{jet})}=\int_{s}^{s / \bar{N}^{2}} \frac{d q^{2}}{q^{2}} \alpha_{s}\left(q^{2}\right)\left[-\frac{C_{c}}{2 \pi} \log \left(\frac{p_{T}^{2} R^{2}}{s}\right)\right],
$$

where $\bar{N} \equiv N \mathrm{e}^{\gamma_{E}}$ with the Euler constant $\gamma_{E}$, and where $C_{c}$ denotes the color charge of parton $c, C_{q}=C_{F}$ for a quark and $C_{g}=C_{A}$ for a gluon. As expected, $J_{c}^{\text {(jet) }}$ is a function of $R$ in this scheme.

The function $\Delta_{a b \rightarrow c d}^{(\mathrm{int})}(N, v)$ is obtained as a trace in color space over hard, soft, and anomalous dimension matrices [5]. All details have been given in [6] and need not be repeated here. The function contributes at NLL level and is the only function in the resummed expression that carries explicit dependence on $v$.

Finally, $\Delta_{c}^{(\mathrm{ng})}(N)$ in (7) contains the contributions from nonglobal logarithms. These were shown [15] to arise when an observable is sensitive to radiation in only a part of phase space, as is the case for a jet defined by some jet "size" parameter $R$. Their resummation is highly nontrivial. Nonglobal logarithms for jet production first enter as a term $\propto[\log (z) / z]_{+}$in $\omega_{a b}^{(2)}$. As discussed in [16], the nonglobal terms arise independently from the boundary of each individual (narrow) "observed" jet. The appropriate second-order coefficient for our case of a single-inclusive jet cross section may, therefore, be directly obtained from $[15,16]$, adjusting the argument of the logarithm properly. We note that these considerations - and in fact the general structure of our resummed cross section-apply to the anti$k_{t}$ algorithm [16]. Finally, we also mention that the nonglobal component makes a rather small contribution (a few percent) to our numerical NNLO results presented below. All in all, after performing the Mellin inverse to $z$ space, the two-loop expansion of the product $\Delta_{a b \rightarrow c d}^{(\mathrm{int})}(N, v) \Delta_{c}^{(\mathrm{ng})}(N)$ in Eq. (7) takes the form

$$
\begin{aligned}
& \left(\frac{\alpha_{s}}{\pi}\right)^{2}\left\{\tilde{\omega}_{a b}^{(0)}(v)\left[\delta(z)+\frac{1}{2}\left(\frac{\alpha_{s}}{\pi}\right)^{2} \mathcal{C}_{c}^{(\mathrm{ng})}\left(\frac{\log (z)}{z}\right)_{+}\right]\right. \\
& +\frac{\alpha_{s}}{\pi}\left[\mathcal{T}_{a b \rightarrow c d}(v) \delta(z)+\mathcal{G}_{a b \rightarrow c d}^{(1)}(v)\left(\frac{1}{z}\right)_{+}\right] \\
& \left.+\left(\frac{\alpha_{s}}{\pi}\right)^{2} \mathcal{G}_{a b \rightarrow c d}^{(2)}(v)\left(\frac{\log (z)}{z}\right)_{+}\right\},
\end{aligned}
$$

with $\mathcal{C}_{c}^{(\mathrm{ng})}=-C_{A} C_{c} \pi^{2} / 3$ for the coefficient of the nonglobal term. The coefficients $\mathcal{G}_{a b \rightarrow c d}^{(1)}(v)$ are predicted by the resummation formalism. The coefficients $\mathcal{T}_{a b \rightarrow c d}(v)$ may be derived by comparison to the explicit NLO results of [11] in the narrow-jet approximation. Along with the known resummation coefficients, knowledge of the $\mathcal{T}_{a b \rightarrow c d}(v)$ is sufficient for determining $\mathcal{G}_{a b \rightarrow c d}^{(2)}(v)$ $[17,18]$. In this way, combining with the contributions from $\Delta_{a}, \Delta_{b}, J_{c}^{\text {(jet) }}, J_{d}^{\text {(recoil) }}$, we obtain full control over the terms $\propto\left(\log ^{3}(z) / z\right)_{+},\left(\log ^{2}(z) / z\right)_{+},(\log (z) / z)_{+}$in $\omega_{a b}^{(2)}$.

Phenomenological results and discussion.-Figure 1 shows results for the differential single-inclusive jet cross section at the LHC, at lowest order as well as for the NLO

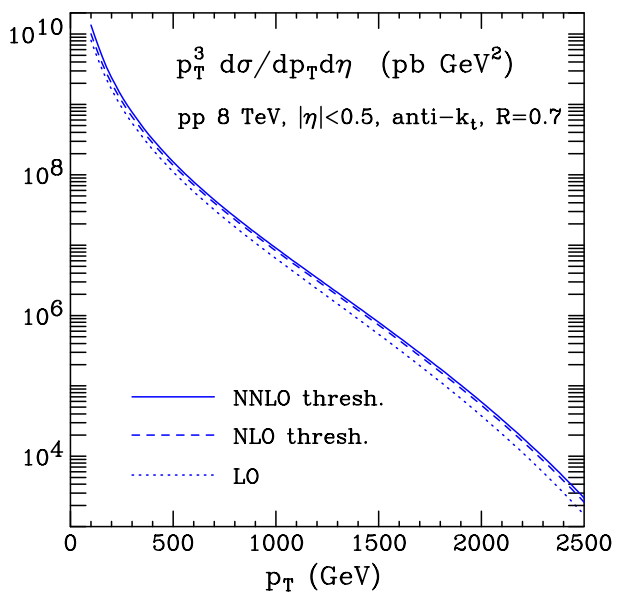

FIG. 1 (color online). Differential cross section for jet production in $p p$ collisions at the LHC at $\sqrt{S}=8 \mathrm{TeV}$, using the anti- $k_{t}$ algorithm with $R=0.7$. 


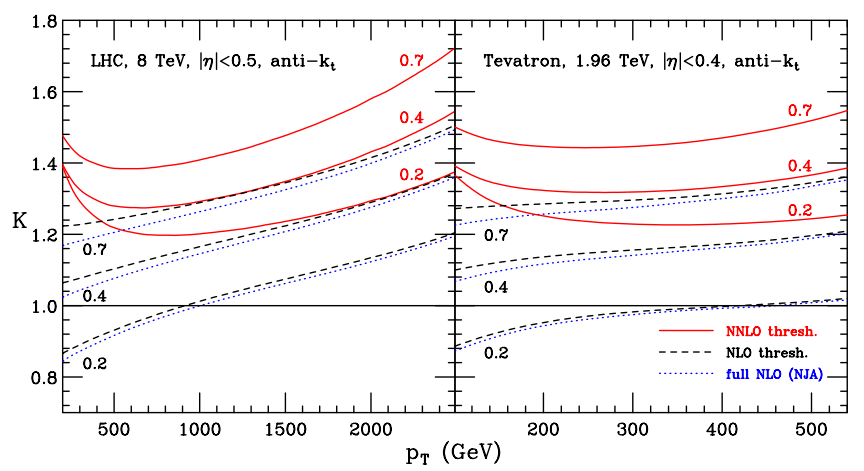

FIG. 2 (color online). Left: $K$ factors for jet production in $p p$ collisions at the LHC at $\sqrt{S}=8 \mathrm{TeV}$ for $R=0.2,0.4,0.7$, using the anti- $k_{t}$ algorithm. Right: Same for $p \bar{p}$ collisions at the Tevatron at $\sqrt{S}=1.96 \mathrm{TeV}$.

and NNLO threshold terms. Here we use the CTEQ6.6 [19] parton distribution functions and scale $\mu=p_{T}$. The left part of Fig. 2 displays the corresponding " $K$-factors," defined as ratios of higher-order cross sections over the leading-order (LO) one, while the right part of the figure is for $p \bar{p}$ collisions at Tevatron at $\sqrt{S}=1.96 \mathrm{TeV}$. Results are presented for various jet parameters $R$. The dotted lines show the NLO results of [11] which were obtained in the NJA for the anti- $k_{t}$ algorithm. We note that these agree with the NLO ones by the "FASTJET" code [9] (as shown in [7]) to better than 3\%, even at $R=0.7$. The dashed lines present the results for the NLO expansion of the threshold terms. It is evident that the latter provide a very faithful description of the full NLO results for much of the $p_{T}$ ranges relevant at LHC and Tevatron. This holds true for each value of $R$, thanks to the fact that the threshold logarithms carry $R$ dependence in our approach, in contrast to that in [6,7]. Finally, the solid lines display the approximate NNLO results. These show a striking further increase of the jet cross sections as compared to NLO, particularly so at high $p_{T}$ where the threshold terms are expected to dominate.

Given the large size of the NNLO corrections observed in Fig. 2, it is, of course, crucial to verify that the predicted enhancements are realistic. Fortunately, recently a full NNLO calculation for jet production in the "gluon-only" channel was presented $[3,4]$, corresponding to $g g$ scattering and to setting the number of flavors $N_{f}=0$ in the partonic matrix elements. It is straightforward to compute our threshold terms in this limit. The comparison is shown in Fig. 3. One can see that the large enhancement at high $p_{T}$ predicted by the NNLO threshold terms is very nicely consistent with the full result. Judging from the comparison, the NNLO threshold terms become accurate at about $p_{T}=400 \mathrm{GeV}$ for the chosen rapidity interval. Additional comparisons with the results of [4] show that this value is representative of rapidity intervals that contain the dominant region $\eta \approx 0$. One also finds that at very forward rapidities, $\eta \sim 4$, our results indicate substantial NNLO $K$

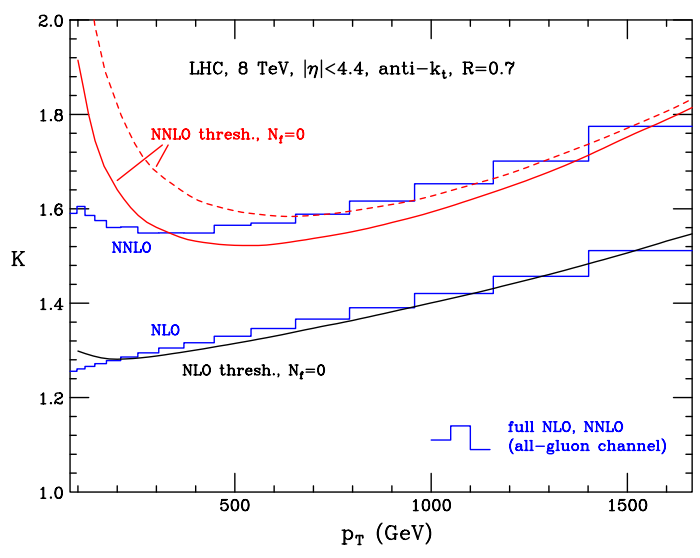

FIG. 3 (color online). $K$ factors for jet production in $p p$ collisions at the LHC at $\sqrt{S}=8 \mathrm{TeV}$ in the "gluon-only" channel. The anti- $k_{t}$ algorithm with $R=0.7$ was used and the NNLO parton distributions of [20]. The histograms show the results of the recent full NNLO calculation [4] and its NLO counterpart, while the lines display the NLO and NNLO threshold terms.

factors of order 5 or so at $p_{T} \sim 40 \mathrm{GeV}$. This, again, appears to be consistent with the results shown in [4]. In this regime, the coefficients of the threshold logarithms become large, due to "small- $x$ " $t$-channel gluon exchange contributions. It will be important for future work to address this region in more detail in order to derive reliable predictions for the forward jet cross section at the LHC. Such contributions may also be responsible in part for the rise of the $K$ factor toward lower $p_{T}$. This rise is more pronounced for the NNLO threshold terms, implying that subleading contributions become relevant here. Whether these are related to subleading logarithmic terms, or to terms that vanish at partonic threshold $z=0$, will need to be studied in more detail. In order to shed light on terms of the latter type, the dashed line in Fig. 3 shows the NNLO threshold result found when using a different angular variable, $v^{\prime} \equiv 1+t / s=z+v(1-z)$, in Eq. (1). Clearly, $v^{\prime}=v+\mathcal{O}(z)$. The difference between the two NNLO threshold results indicates a typical uncertainty of the prediction obtained from threshold resummation.

We thank A. Gehrmann-De Ridder, M. Klasen, and $\mathrm{S}$. Moch for useful communications. We are grateful to M. Kumar for pointing out an inconsistency in our initial numerical results, and to J. Rojo for reporting detailed numerical studies obtained with our code. A. M. thanks the Alexander von Humboldt Foundation, Germany, for support through a Fellowship for Experienced Researchers.

[1] C. Meyer (ATLAS and CMS Collaborations), arXiv:1310.2946, and references therein.

[2] C. Mesropian (CDF and D0 Collaborations), arXiv:1106.3119, and references therein. 
[3] A. Gehrmann-De Ridder, T. Gehrmann, E. W. N. Glover, and J. Pires, Phys. Rev. Lett. 110, 162003 (2013).

[4] J. Currie, A. Gehrmann-De Ridder, E. W. N. Glover, and J. Pires, J. High Energy Phys. 01 (2014) 110. See the references for an extensive list of previous perturbative jet calculations.

[5] N. Kidonakis, G. Oderda, and G. F. Sterman, Nucl. Phys. B525, 299 (1998).

[6] N. Kidonakis and J. F. Owens, Phys. Rev. D 63, 054019(2001).

[7] M. C. Kumar and S.-O. Moch, arXiv:1309.5311.

[8] See also: M. Klasen, G. Kramer, and M. Michael, arXiv:1310.1724.

[9] M. Cacciari, G. P. Salam, and G. Soyez, Eur. Phys. J. C 72, 1896 (2012).

[10] B. Jäger, M. Stratmann, and W. Vogelsang, Phys. Rev. D 70, 034010 (2004).

[11] A. Mukherjee and W. Vogelsang, Phys. Rev. D 86, 094009 (2012).

[12] D. de Florian and W. Vogelsang, Phys. Rev. D 76, 074031 (2007).
[13] M. Cacciari, G. P. Salam, and G. Soyez, J. High Energy Phys. 04 (2008) 063.

[14] S. Catani, M. Grazzini, and A. Torre, Nucl. Phys. B874, 720 (2013).

[15] M. Dasgupta and G. P. Salam, Phys. Lett. B 512, 323 (2001); A. Banfi and M. Dasgupta, J. High Energy Phys. 01 (2004) 027.

[16] A. Banfi, M. Dasgupta, K. Khelifa-Kerfa, and S. Marzani, J. High Energy Phys. 08 (2010) 064.

[17] N. Kidonakis, E. Laenen, S. Moch, and R. Vogt, Phys. Rev. D 64, 114001 (2001).

[18] L. G. Almeida, G. F. Sterman, and W. Vogelsang, Phys. Rev. D 80, 074016 (2009).

[19] P. Nadolsky, H.-L. Lai, Q.-H. Cao, J. Huston, J. Pumplin, D. Stump, W.-K. Tung, and C.-P. Yuan, Phys. Rev. D 78, 013004 (2008).

[20] A. D. Martin, W. J. Stirling, R. S. Thorne, and G. Watt, Eur. Phys. J. C 63, 189 (2009). 